\title{
Dissociable Effects of Lesions to Orbitofrontal Cortex Subregions on Impulsive Choice in the Rat
}

\author{
Adam C. Mar, Alice L. J. Walker, David E. Theobald, Dawn M. Eagle, and Trevor W. Robbins \\ Behavioural and Clinical Neuroscience Institute, Department of Experimental Psychology, University of Cambridge, Cambridge CB2 3EB, United Kingdom
}

The orbitofrontal cortex $(\mathrm{OFC})$ is implicated in a variety of adaptive decision-making processes. Human studies suggest that there is a functional dissociation between medial and lateral OFC (mOFC and 1OFC, respectively) subregions when performing certain choice procedures. However, little work has examined the functional consequences of manipulations of OFC subregions on decision making in rodents. In the present experiments, impulsive choice was assessed by evaluating intolerance to delayed, but economically optimal, reward options using a delay-discounting paradigm. Following initial delay-discounting training, rats received bilateral neurotoxic or sham lesions targeting whole OFC (wOFC) or restricted to either mOFC or 10FC subregions. A transient flattening of delay-discounting curves was observed in wOFC-lesioned animals relative to shams- differences that disappeared with further training. Stable, dissociable effects were found when lesions were restricted to OFC subregions; mOFC-lesioned rats showed increased, whereas lOFC-lesioned rats showed decreased, preference for the larger-delayed reward relative to sham-controls-a pattern that remained significant during retraining after all delays were removed. When locations of levers leading to small-immediate versus large-delayed rewards were reversed, wOFC- and 10FC-lesioned rats showed retarded, whereas mOFC-lesioned rats showed accelerated, trajectories for reversal of lever preference. These results provide the first direct evidence for dissociable functional roles of the m0FC and $10 \mathrm{FC}$ for impulsive choice in rodents. The findings are consistent with recent human functional imaging studies and suggest that functions of mOFC and $10 \mathrm{FC}$ subregions may be evolutionarily conserved and contribute differentially to decision-making processes.

\section{Introduction}

The orbitofrontal cortex (OFC) has been strongly implicated in reinforcement-guided decision making (Murray et al., 2007; Wallis, 2007; Doya, 2008). OFC damage in humans leads to increased impulsivity, which may result from bias toward immediate rewards (Bechara et al., 2000; Berlin et al., 2004). Rodent studies have documented similar effects using delay-discounting paradigms: when offered the choice between smaller, moreimmediate or larger, more-delayed rewards, OFC-lesioned animals tend to choose impulsively, i.e., the smaller, moreimmediate option (Mobini et al., 2002; Rudebeck et al., 2006). However, other studies have reported that OFC lesions can shift preference toward the less-impulsive, delayed-reward alternative (Kheramin et al., 2002; Winstanley et al., 2004).

Two main hypotheses have been advanced to explain these contrasting findings. The first proposes the OFC to be involved in flexibly updating the incentive value of outcomes, whereby OFC

Received Dec. 18, 2010; revised March 4, 2011; accepted March 13, 2011.

Author contributions: A.C.M. designed research; A.C.M., A.L.J.W., and D.E.T. performed research; D.M.E. and T.W.R. contributed unpublished reagents/analytic tools; A.C.M. analyzed data; A.C.M. and T.W.R. wrote the paper.

This work was supported by Wellcome Trust Grant 089589/Z/09/Z to T.W.R, B. J. Everitt, A.C. Roberts, J.W. Dalley, and B. J. Sahakian. The Behavioural and Clinical Neuroscience Institute is cofunded by the Medical Research Council and the Wellcome Trust. We thank Alan Lyon for his help with the histological procedures. Parts of this paper have been presented previously in abstract form (Society for Neuroscience 2008, 34:876.14).

This article is freely available online through the J Neurosci Open Choice option.

Correspondence should be addressed to Adam C. Mar, Behavioural and Clinical Neuroscience Institute, Department of Experimental Psychology, Downing Street, University of Cambridge, Cambridge CB2 3EB, UK. E-mail: am682@cam.ac.uk.

DOI:10.1523/JNEUROSCI.6620-10.2011

Copyright $\odot 2011$ the authors $\quad 0270-6474 / 11 / 316398-07 \$ 15.00 / 0$ damage may increase within-session perseverative responding (Winstanley et al., 2004; Schoenbaum and Roesch, 2005; Rudebeck et al., 2006). The second hypothesis emphasizes possible functional heterogeneity within the OFC. For instance, quantitative analysis of intertemporal choice data in OFC-lesioned rats has implicated two processes underlying OFC function-an increased sensitivity to the relative value of the larger of two reinforcers and an increased rate of delay discounting - which may augment or diminish impulsive choice depending on the magnitudes of, and delays to, reward options (Kheramin et al., 2002). Electrophysiological studies have identified two distinct populations of single-unit firing patterns within rat OFC in anticipation of impending reward: a subset that maintains activity across delays and a subset that decreases activity across delays and correlates with discounting behavior (Roesch et al., 2006). Moreover, different subregions of the OFC may have different functions. In primates, medial OFC ( $\mathrm{mOFC}$ ) has been associated with responding to, monitoring, or adjusting the incentive value of stimuli, whereas lateral OFC (lOFC) has been implicated in punishment or suppression of previously rewarded responses following contingency change (Iversen and Mishkin, 1970; Elliott et al., 2000; O’Doherty et al., 2001; Kringelbach and Rolls, 2004). Human functional imaging experiments have correlated the degree of activation in lateral prefrontal (including lOFC) areas with choice of delayed rewards, whereas activity in limbic regions such as ventral striatum and mOFC have been correlated with choice of relatively immediate rewards (McClure et al., 2004, 2007).

The present experiments sought to test these hypotheses and further clarify the role of the OFC in delay-discounting in the rat. 
A
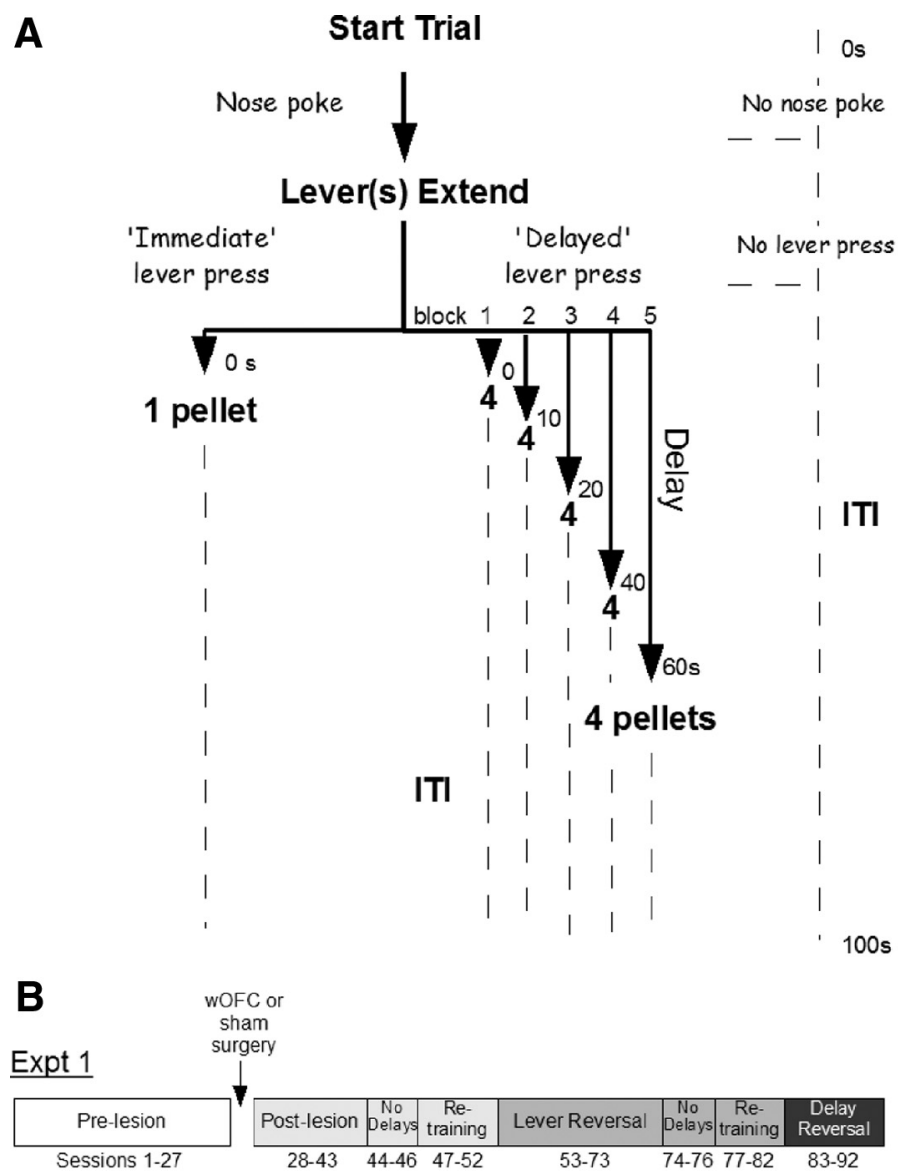

\section{Expt 2}

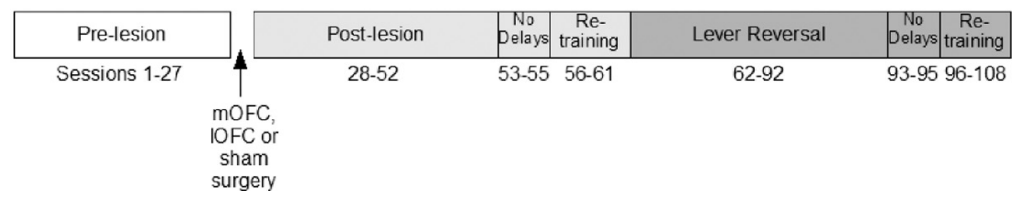

Figure 1. Outline of delay-discounting task. $\boldsymbol{A}$, Basic structure of individual trials. $\boldsymbol{B}$, Timeline of experimental manipulations. Postlesion and lever-reversal phases were extended in experiment (Expt) 2 to ensure reliability of significant effects.

First, we assessed whether patterns of delay-discounting behavior following neurotoxic lesions targeting whole OFC (wOFC) are related to reductions in flexible responding by reversing the spatial location of small-immediate versus large-delayed operant choices, and by reversing the order of delay presentation. Second, we examined the putative dissociable effects of more selective mOFC or lOFC lesions on delay-discounting performance, where it was hypothesized that mOFC lesions preferentially affect processing of more-immediate, whereas lOFC lesions preferentially affect processing of more-delayed, rewards.

\section{Materials and Methods}

Subjects. Male Lister Hooded rats (Charles River), weighing 250-325 g at the start of each experiment, were maintained at $85-90 \%$ of their freefeeding weight. Water was available ad libitum. Animals were housed 2-4 per cage under reversed light/dark cycle (light-phase 19:00 h-07:00 h) with behavioral testing performed between 08:00 h and 19:00 h. Experiments were undertaken in accordance with the UK Animals (Scientific Procedures) Act, 1986.

Behavioral testing. The apparatus and testing procedure have been described previously (Cardinal et al., 2000; Mar and Robbins, 2007). Testing occurred in eight operant-conditioning chambers (Med Associates) equipped with a house light, two retractable levers located on either side of a centrally positioned receptacle into which a dispenser delivered $45 \mathrm{mg}$ food pellets (TestDiet; Purina), and a receptacle light. Receptacle head-entries were detected by an infrared photodiode. The apparatus was controlled using software written by Rudolf N. Cardinal in Arachnid (experiment 1) and using the WhiskerControl system (Cardinal and Aitkin, 2010) (experiment 2).

Subjects were first trained to press either the left or right lever to obtain a food pellet, and subsequently to nose-poke into the receptacle to trigger lever presentation in discrete trials every $40 \mathrm{~s}$ [session-performance criteria of 60 rewarded trials ( 30 per lever)]. Rats were then trained on the delay-discounting procedure (Fig $1 A$ ). Each session comprised 60 trials (five 12-trial blocks). Each block began with two forced trials in which only one lever was presented (randomly left or right per trial pair), followed by 10 choice trials in which both levers were presented. Each rat had one lever designated (counterbalanced) as immediate (associated with one, immediate pellet) and one lever as delay [associated with four pellets, delivered after a delay that was increased progressively $(0,10,20,40,60 \mathrm{~s})$ between successive blocks]. Trial onset was signaled every $100 \mathrm{~s}$ by illumination of the house and receptacle lights, after which rats had to nose-poke into the receptacle to trigger lever presentation. Failures to nose-poke or to lever-press within $10 \mathrm{~s}$ were deemed omissions and the chamber reset to the intertrial interval (ITI) state-all lights extinguished and levers retracted-until the next trial. Following lever-presses, levers were retracted and the house light extinguished. Reward delivery coincided with $6 \mathrm{~s}$ illumination of the receptacle light followed by the ITI state. Figure $1 B$ shows the experimental timeline of delay-discounting sessions for experiments 1 and 2 .

Surgery. In experiment 1,22 rats were allocated to two groups matched for delay-discounting performance. Rats were anesthetized using $100 \mathrm{ml} / \mathrm{kg}$ Avertin [10 g of 2,2,2-tribromoethanol (Sigma) in $5 \mathrm{~g}$ of tertiary amyl alcohol, diluted in $40 \mathrm{ml}$ of ethanol and $450 \mathrm{ml}$ of PBS] and secured in a stereotaxic frame (David Kopf Instruments). Excitotoxic lesions targeting wOFC $(n=13)$ were made using $0.09 \mathrm{M}$ quinolinic acid dissolved in $0.1 \mathrm{M}$ phosphate buffer (vehicle), with $\mathrm{pH}$ adjusted to 7.4 using $0.1 \mathrm{M} \mathrm{NaOH}$. Bilateral infusions were made via 31 gauge stainless-steel injector (Coopers Needleworks) attached to a Hamilton microinfusion pump by polyethylene tubing according to the following parameters: Site 1: anteroposterior $(\mathrm{AP})+4.0 \mathrm{~mm}$, mediolateral $(\mathrm{ML}) \pm 0.8 \mathrm{~mm}$, dorsoventral (DV) $-3.4 \mathrm{~mm}, 0.2 \mu \mathrm{l}$ over $2 \mathrm{~min}$; Site 2 : AP $+3.7 \mathrm{~mm}, \mathrm{ML} \pm 2.0 \mathrm{~mm}$, DV $-3.6 \mathrm{~mm}, 0.3 \mu \mathrm{l}$ over $3 \mathrm{~min}$; Site $3: \mathrm{AP}+3.2 \mathrm{~mm}, \mathrm{ML} \pm 2.6 \mathrm{~mm}$, DV $-4.4 \mathrm{~mm}, 0.3 \mu \mathrm{l}$ over $3 \mathrm{~min}$. The AP, ML, and DV coordinates were taken from bregma, midline, and dura, respectively; the incisor bar was $3.3 \mathrm{~mm}$ below the interaural line. Injectors were left in place for a time period equivalent to the duration of infusion before removal. Sham surgeries $(n=9)$ were performed in similar manner, but with infusing vehicle alone.

In experiment 2, 32 rats were allocated to three groups matched for delay-discounting performance, and received bilateral lesions of mOFC $(n=11), \operatorname{lOFC}(n=11)$, or sham surgery $(n=10 ; 5 \mathrm{mOFC}$ and 5 lOFC). The apparatus and procedures were similar to those in experiment 1 . Excitotoxic lesions were made using $0.1 \mathrm{~m}$ NMDA dissolved in $0.1 \mathrm{M}$ 
phosphate buffer (vehicle), with $\mathrm{pH}$ adjusted to 7.4. Infusions were made using the following parameters (Fuchs et al., 2004): $\mathrm{mOFC:} \mathrm{AP}+4.2$ $\mathrm{mm}, \mathrm{ML} \pm 0.6 \mathrm{~mm}, \mathrm{DV}-4.3 \mathrm{~mm}, 0.2 \mu \mathrm{l}$ over $2 \mathrm{~min}$; lOFC: AP $+3.2 \mathrm{~mm}, \mathrm{ML} \pm 2.5 \mathrm{~mm}$, DV $-3.6 \mathrm{~mm}, 0.3 \mu \mathrm{l}$ over $3 \mathrm{~min}$.

Data analysis. Choice data were analyzed using two complementary logistic regression approaches (Hosmer and Lemeshow, 2000). First, generalized estimating equation (GEE) models with logit link and binomial variance distribution functions were fit to the binarycoded choice data $(0=$ immediate, $1=$ delay $)$ with lesion (mOFC, lOFC, wOFC, or sham), delay $(0,10,20,40,60 \mathrm{~s})$, and their interaction included as categorical predictors, and presurgical choice performance as continuous covariate (to account for baseline differences). Different working correlation specifications (e.g., independence, autoregressive, exchangeable) were assessed to ensure parameter estimate consistency. Jackknife resampling variance estimates were used to construct 95\% confidence intervals and $\chi^{2}$ tests used to assess significance. Significant lesion and lesion $\times$ delay interactions were decomposed using simple logistic regression and/or Bonferronicorrected pairwise comparisons. Second, to reinforce and extend GEE analysis (cf., Bezzina et al., 2007), choice data were fit (using generalized nonlinear mixed-effect models) by a twoparameter logistic equation: \%immediate $=$ $1 /\left[1+(\text { delay/D50) })^{\epsilon}\right]$, where D50 estimates the indifference delay (i.e., $50 \%$ choice for each lever) and $\epsilon$ defines the function's slope. Maximum likelihood estimates of parameters were compared between lesion groups using likelihood ratio (LR) tests. When locations of immediate and delay levers were reversed, delay-discounting behavior was initially disrupted. Thus, speed and extent of reversal learning was analyzed by GEE on total choice preference per three-session block. Statistical analyses were conducted using R (v2.12.0; http://www.r-project.org).

Histology. Rats were terminally anesthetized with sodium pentobarbitone and perfused transcardially with $0.01 \mathrm{~m}$ PBS followed by formaldehyde solution (4\% paraformaldehyde in PBS). Brains were removed, postfixed in formaldehyde solution, and transferred into $20 \%$ sucrose in 0.01 м PBS $24 \mathrm{~h}$ before sectioning on a freezing microtome. Coronal sections $(60 \mu \mathrm{m})$ were stained with cresyl violet and lesion locations were mapped onto standardized sections of the rat brain (Paxinos and Watson, 1998).

\section{Results}

\section{Lesion analysis}

Histological verification of lesions (Fig 2) was conducted by A.C.M. and confirmed by individuals blind to the experimental results. In experiment 1 , three animals were excluded from the wOFC-lesion group: two had incomplete/unilateral lesions and one developed seizures following surgery. wOFC lesions encompassed the OFC subregions delineated by Paxinos and Watson (1998), with some sparing of anterior dorsolateral OFC and of posterior portions of $\mathrm{mOFC}$ and ventral OFC subregions (Fig

A

MOFC

B

$+4.2$

$+5.2$

$+4.7$

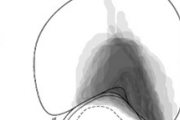

$+3.7$

$+3.2$
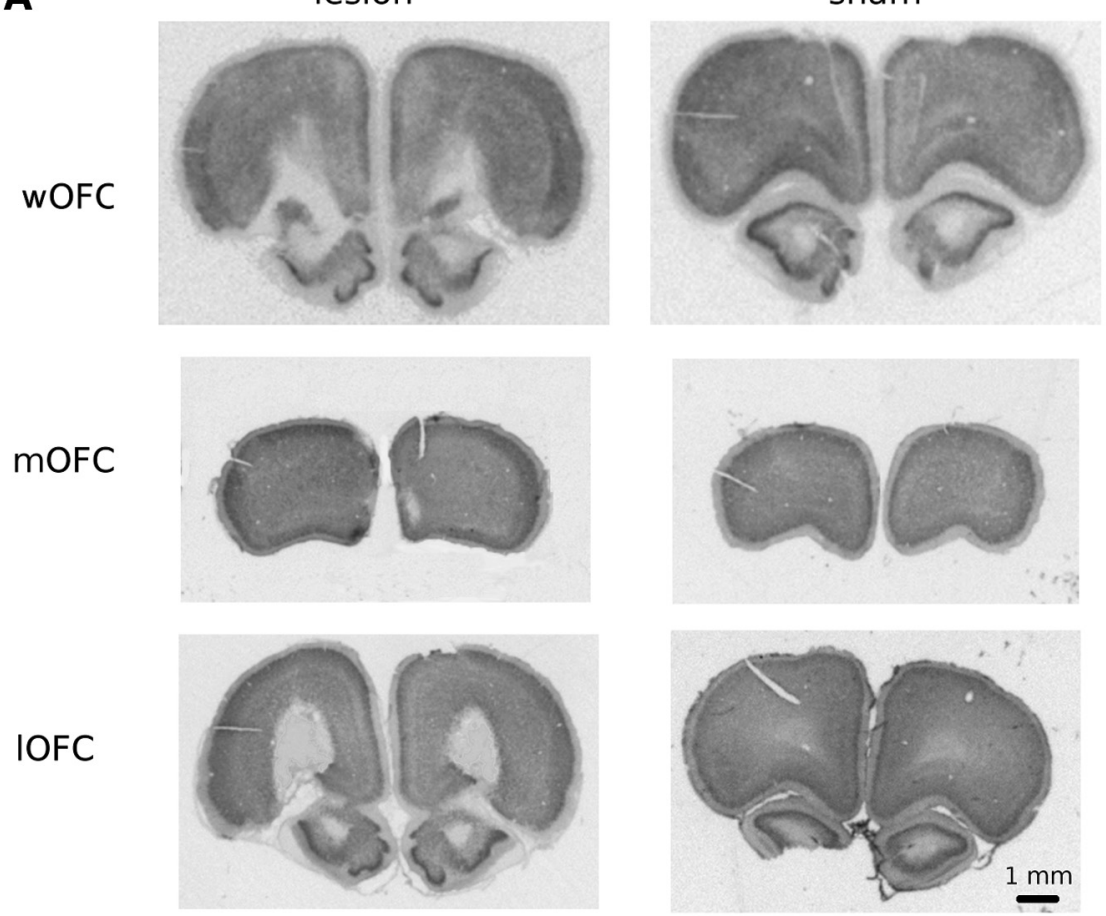

wOFC

MOFC

IOFC
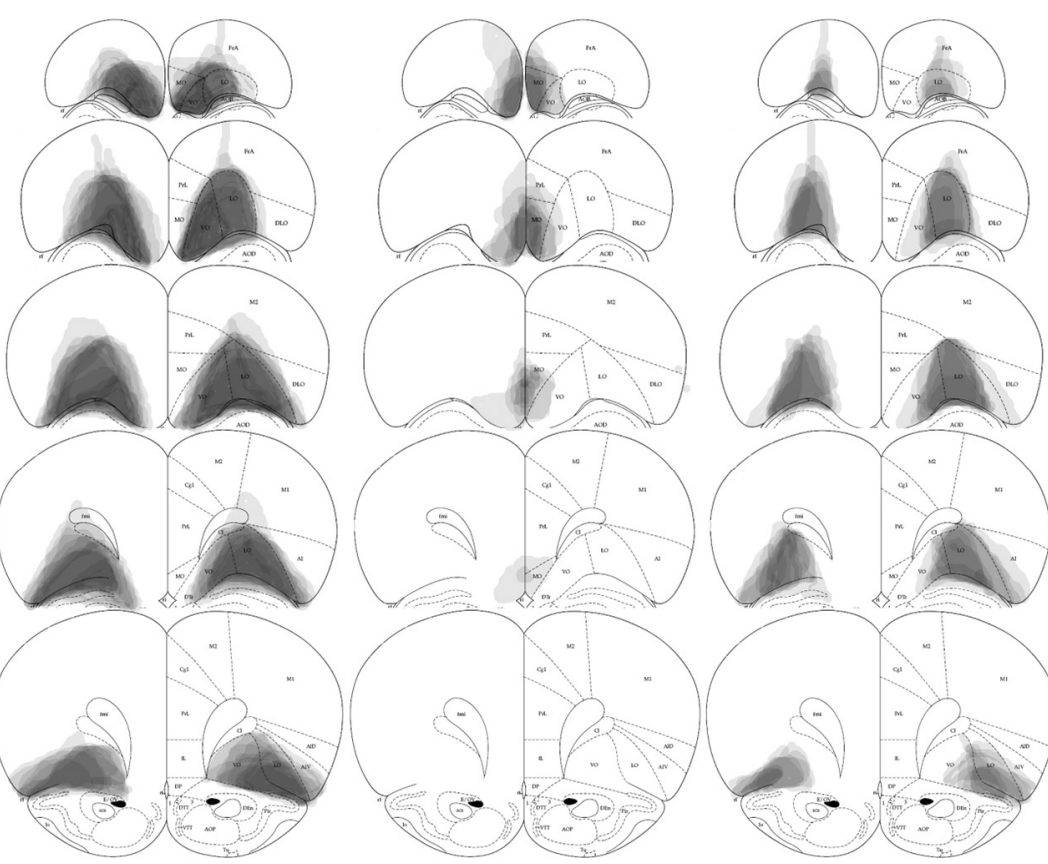

Figure 2. Histological analysis of OFC lesions. $\boldsymbol{A}$, Photomicrographs depicting typical lesions of whole (top left), medial (middle left), and lateral (bottom left) w0F(-operated relative to sham-operated control tissue (right). $\boldsymbol{B}$, Schematic depicting lesion assessments for whole (left), medial (center), and lateral (right) OFC. Grey scale shading indicates extent of neuronal loss across subjects, with each subject represented as a separate, stacked layer. Diagrams are modified from Paxinos and Watson (1998).

$2 B)$. Damage occasionally extended into ventral anterior insula and dorsal edge of accessory olfactory areas. In experiment 2 , five rats were excluded: three had incomplete/unilateral lesions (two mOFC, one lOFC) and two (one mOFC, one lOFC) developed postsurgical seizures. mOFC damage encompassed the mOFC subregion, was typically associated with tissue retraction along the ventromedial surface (Fig 2A), and showed some sparing in 


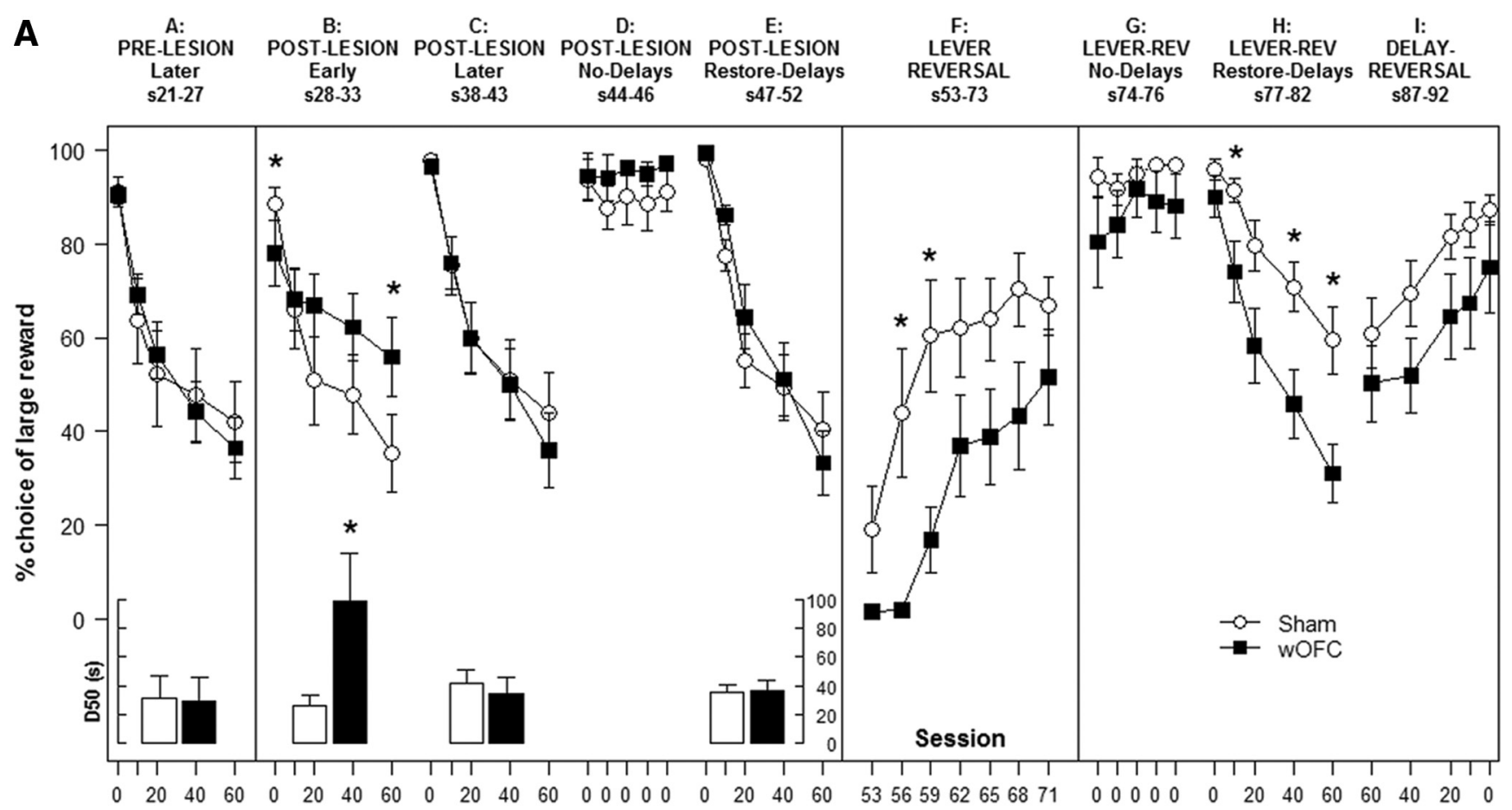

Delay to large reward (s)
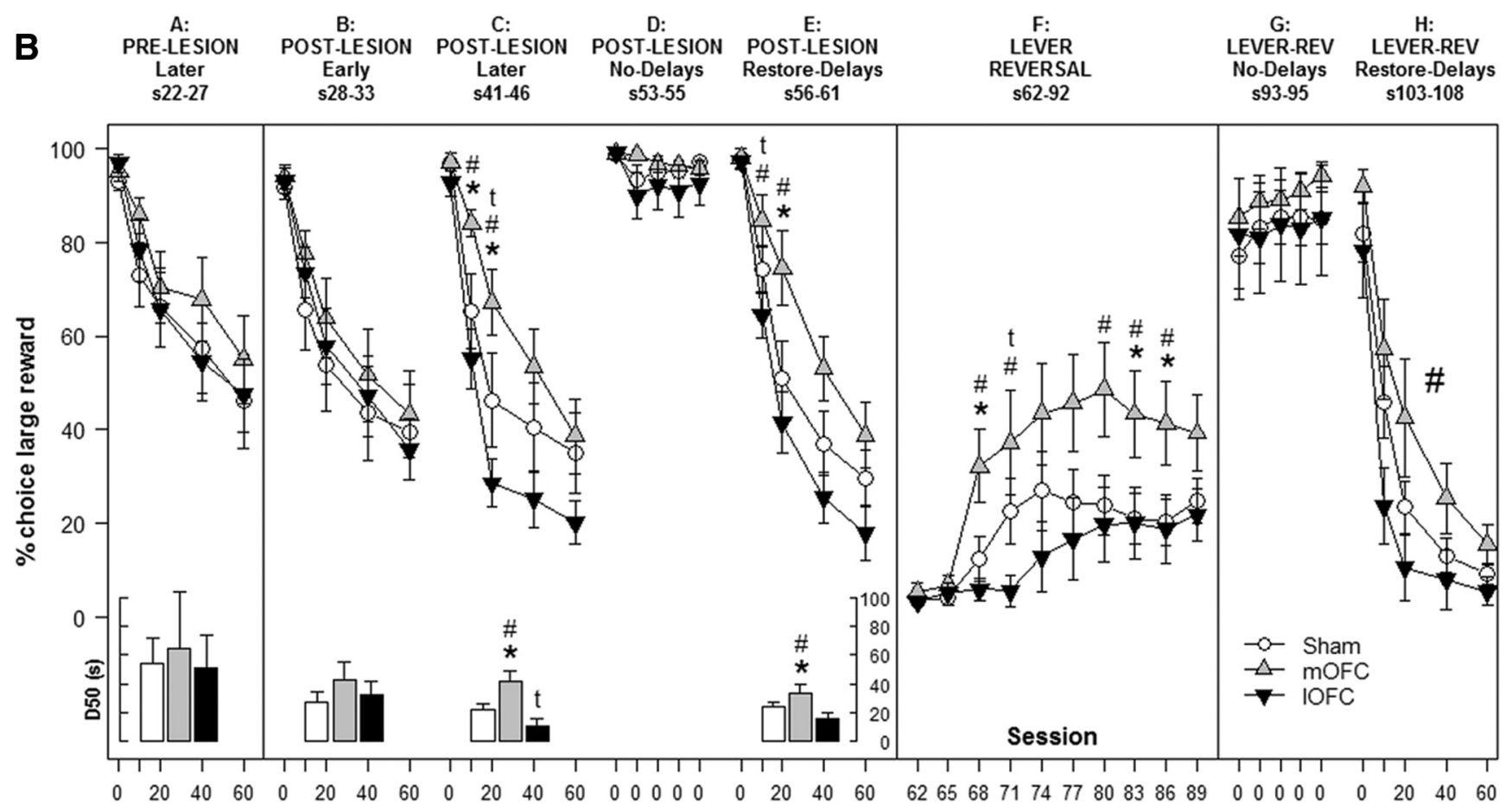

Delay to large reward (s)

Figure 3. Effect of OFC lesions on delay discounting. $\boldsymbol{A}, \boldsymbol{B}$, Mean ( + SEM) percentage choice of the large reward across delays for wOFC and sham-operated controls ( $\boldsymbol{A})$ and for $\mathrm{m} 0 \mathrm{FC}, \mathrm{IOFC}$, and sham-operated controls $(\boldsymbol{B})$. Column F displays the mean ( + SEM) percentage choice for the large reward for three-session blocks following reversal of lever positions. Inset depicts fitted indifference delay parameter (D50) for each group per corresponding phase. ${ }^{*} p<0.05 \mathrm{WOFC}(\boldsymbol{A})$ or m0FC (B) versus sham. ${ }^{\mathrm{t}} p<0.05 \mathrm{IOFC}$ versus sham. ${ }^{\#} p<0.05 \mathrm{~m} 0 \mathrm{FC}$ versus IOFC (large symbol, main effect).

posterior sections (Fig $2 \mathrm{~B}$ ). lOFC damage encompassed the lOFC subregion, occasionally extending laterally into edges of the adjacent ventral OFC and/or dorsolateral OFC, with some small sparing in the anterior and posterior limits (Fig $2 B$ ).
Experiment 1: Whole OFC lesions

Logistic GEE model analysis revealed that, preoperatively, rats exhibited significant delay-discounting $\left[\chi^{2}(4)=16.6, p<\right.$ $0.01]$, with no differences between performance-matched groups 
(Fig 3A, column A). Discounting curves of wOFC-lesioned rats appeared flattened in the early postoperative period (Fig $3 A$, column $B)$, confirmed by a significant lesion $X$ delay interaction $\left[\chi^{2}(4)=10.8, p<0.05\right]$ in which wOFC-lesioned rats exhibited decreased preference for the large reward at 0 s delay $(p<0.05)$, but increased preference at the $60 \mathrm{~s}$ delay $(p<0.05)$, relative to shams. The lesion-group differences disappeared after further training (Fig $3 A$, column $\mathrm{C}$ ). When delays to the large reward were removed, all rats shifted their behavior to consistently choose the large reward (Fig $3 A$, column D). When delays were reintroduced (Fig $3 A$, column E), delay-discounting was reestablished $\left[\chi^{2}(4)=17.0, p<0.01\right]$ but with no significant differences between wOFC- and sham-lesioned animals.

These results were supported by fitting logistic functions (\%immediate $=\left[1 /\left(1+(\text { delay/D50 })^{\epsilon}\right]\right)$ to the delay-discounting data. wOFC lesions increased D50 (Fig. $3 A$, bar graphs) $\left[\operatorname{LR} \chi^{2}(1)=4.6\right.$, $p<0.05$ ], which disappeared with further training and following removal and restoration of delays. No differences in the slope parameter, $\epsilon$, were observed.

When positions of the levers leading to small-immediate and large-delayed rewards were switched (Fig 3A, column F), wOFClesioned rats showed a reduction in choice-preference for the larger reward $\left[\chi^{2}(19)=57.14, p<0.001\right]$ from sessions $4-9$ $(p s<0.05)$ relative to shams. When delays were subsequently removed, all rats shifted their behavior to consistently choose the large reward (Fig $3 A$, column $\mathrm{G}$ ). On restoration of the delays (Fig $3 A$, column $\mathrm{H})$, all rats reestablished delay-discounting $\left[\chi^{2}(4)=\right.$ 15.4, $p<0.01$ ], however, wOFC-lesioned animals showed increased impulsive choice relative to sham-operated controls $\left[\chi^{2}(4)=12.6, p<0.05\right]$ at 10,40 , and 60 s delays $(p s<0.05)$. When the order of delays to the large reward was reversed (Fig $3 A$, column I), both wOFC and sham-operated groups shifted their responding to track delays $\left[\chi^{2}(4)=11.6, p<0.05\right]$ but with no significant differences between lesion groups. Trial omissions, lever-press and reward-collection latencies were not significantly different between groups in any experimental phase.

\section{Experiment 2: Medial versus lateral OFC lesions}

Preoperatively, rats exhibited significant delay-discounting $\left[\chi^{2}(4)=21.5, p<0.01\right]$, with no differences between performance-matched groups (Fig 3B, column A). Although no significant lesion effects were observed in the early postlesion period (Fig $3 B$, column B), a significant lesion $\times$ delay interaction for choice behavior $\left[\chi^{2}(8)=18.8, p<0.05\right]$ emerged later (Fig $3 B$, column C). Relative to both sham-operated and lOFClesioned animals, mOFC-lesioned rats exhibited increased preference for the larger reward at 10 and 20 s delays $(p<0.05$ and $p<0.01$, respectively). Relative to shams, 1OFC-lesioned rats showed significantly decreased preference for the larger reward at the $20 \mathrm{~s}$ delay $(p<0.05)$. When all delays were removed, all rats shifted their behavior to consistently choose the large reward (Fig $3 B$, column $\mathrm{D})$. When delays to the large reward were reintroduced (Fig 3B, column E), dissociable preferences were reestablished: a significant lesion $\times$ delay interaction $\left[\chi^{2}(8)=17.6, p<\right.$ 0.05 ] with lOFC significantly decreased compared with sham and $\mathrm{mOFC}$ in preference for the large reward at the $10 \mathrm{~s}$ delay $(p s<$ 0.05 ), and mOFC significantly increased compared with sham and lOFC in preference for the large reward at the $20 \mathrm{~s}$ delay $(p s<0.01)$.

The GEE results were reinforced by fitting logistic functions $\left(\%\right.$ immediate $=\left[1 /\left(1+(\text { delay/D50 })^{\epsilon}\right]\right)$ to the discounting data. Significant differences in the estimated indifference delay, D50, (Fig. 3B, bar graphs) were observed between mOFC-lesion and shams $\left[\operatorname{LR} \chi^{2}(1)=6.7, p<0.01\right]$, lOFC-lesion and shams $\left[\operatorname{LR} \chi^{2}(1)=3.9, p<0.05\right]$, and mOFC-lesion and lOFC-lesion $\left[\operatorname{LR} \chi^{2}(1)=10.5, p<0.01\right]$ in later postsurgical sessions and remained relatively stable following removal and restoration of delays (sham-mOFC: $\operatorname{LR}^{2}(1)=8.8, p<0.01$; sham-lOFC: $\mathrm{LR} \chi^{2}(1)=3.2, p=0.074$; mOFC-lOFC: $\operatorname{LR}^{2}(1)=9.8, p<$ $0.01)$. No differences in slope parameter, $\epsilon$, were observed.

When the positions of the levers leading to small-immediate and large-delayed rewards were switched (Fig $3 B$, column F), a significant lesion $\times$ session interaction was found $\left[\chi^{2}(58)=\right.$ 86.6, $p<0.01$ ], with mOFC-lesioned rats showing faster reversal of choice-preference relative to sham-controls and lOFClesioned animals at sessions 7-9 and 19-24 ( $p$ s $<0.05)$, and lOFC-lesioned rats showing retarded trajectories relative to shams at sessions $10-12(p<0.05)$. When all delays were removed, rats shifted their behavior to consistently choose the large reward (Fig 3B, column G). When delays were reintroduced (Fig $3 B$, column $\mathrm{H}$ ), rats recovered their delay-discounting behavior $\left[\chi^{2}(4)=22.4, p<0.001\right]$ and the difference between lOFC- and mOFC-lesioned rats reemerged as a significant main effect $\left[\chi^{2}(2)=6.9, p<0.05\right]$. Trial omissions, lever press, and rewardcollection latencies were not significantly different between groups in any experimental phase.

\section{Discussion}

This study provides the first demonstration of functionally contrasting effects of selective lesions of medial and lateral subregions of $\mathrm{OFC}$ on delay-discounting performance in the rat; mOFC-lesioned rats exhibited a stable pattern of decreased impulsive choice and lOFC-lesioned rats showed increased impulsive choice, relative to sham-controls. These results may help explain why prior studies of OFC function have produced contradictory findings and are discussed in terms of possible heterogeneity of functions represented within rodent OFC.

\section{Whole OFC}

Reduction in impulsive choice following wOFC lesions replicates Winstanley et al. (2004). However, this pattern disappeared as postsurgical training progressed. Such labile postlesion effects are consistent with prior studies. Rudebeck et al. (2006) found differences in impulsive choice across six sessions following OFC lesions, but not after delays between choice-options were equated. Cardinal et al. (2001) observed a flattening of delaydiscounting curves across seven sessions following medial prefrontal cortex lesions that disappeared when delays were removed in alternating sessions. Moreover, after extended training using task parameters similar to those of the present study (e.g., short delays to small-reward delivery), Kheramin et al. (2002) observed no differences in choice-behavior between OFC- and sham-lesioned rats.

These transient alterations of choice-behavior were unlikely caused by functional recovery, as wOFC-lesioned animals subsequently showed slower reversal of choice-preference after locations of the immediate and delay levers were switched. Disruption of choice-behavior in OFC-lesioned animals after reversal is consistent with numerous studies (Chudasama and Robbins, 2003; McAlonan and Brown, 2003; Boulougouris et al., 2007). The transience of wOFC-lesion discounting effects perhaps reflects disruption of task-related representations, including outcome expectancies, that could be relearned with support from intact network structures such as the basolateral amygdala (BLA) or striatum (Schoenbaum and Roesch, 2005; Schoenbaum et al., 2009). 
Reduced flexibility of wOFC-lesioned animals following lever-location reversal might suggest that their delay-discounting performance is related to within-session perseveration (Winstanley et al., 2004). However, after extended reversal training, wOFC-lesioned rats exhibited increased impulsive choice despite initially (no-delays) choosing the large reward to a similar extent as sham-controls. It is unclear to what extent these postreversal differences in choice-behavior are attributable to residual effects from the reversal phase. Nevertheless, when within-session delays were removed or presented in reverse order, wOFC-lesioned animals shifted their choice-behavior, performing comparably to shams. Thus, although wOFC lesions disrupted aspects of between-session flexibility, such disruptions do not account simply for differences in delay-discounting.

\section{Lateral OFC}

lOFC-lesioned rats increased impulsive choice relative to both sham-controls and mOFC-lesioned animals after several postsurgical training sessions; this effect was still evident after retraining following removal of all delays. This persistent choice-bias fits neatly with human neuroimaging data. The degree of activation of lateral areas of frontal cortex (including lOFC) predicts the extent of deferral of gratification (McClure et al., 2004), correlates negatively with individual differences in relative preference for smaller-immediate rewards (Hariri et al., 2006), and correlates positively with the inclination to wait for larger-delayed rewards (Boettiger et al., 2007). Thus, lOFC may participate in circuitry that mediates cognitive control such as prospective planning or behavioral regulation in the context of stimulusindependent, long-term goals (Windmann et al., 2006).

IOFC is implicated in cognitive control when rewardassociated responses require suppression (Iversen and Mishkin, 1970; Elliott et al., 2000) or when punishment-related stimuli guide behavioral change (O'Doherty et al., 2001). In the context of impulsive choice, resisting selection of immediate rewards may require inhibitory-control and/or negative-feedback mechanisms that are disrupted by lOFC lesions. This interpretation is consistent with our observation that lOFC-lesioned animals show attenuated reversal of choice-preference when locations of immediate and delay levers were switched. The smaller effect size of IOFC lesions relative to wOFC lesions on reversal may relate to smaller lesion size or may have been masked by slower (for unknown reasons) reversal trajectories of sham-controls in experiment 2. Regardless, the similar directionality of 1OFC- and wOFC-lesion effects suggests that lOFC is important for flexibility during reversal learning, consistent with other studies (Schoenbaum et al., 2003; Gourley et al., 2010).

Alternatively, enhanced preference for immediate over delayed rewards in lOFC-lesioned rats may be due to disruption of associative learning processes. The delay between stimulus (or response) and outcome renders representation of their association more difficult, requiring temporal-bridging mechanisms. Roesch et al. (2006) identified cells within lOFC, the activity of which rose until delayed-reward delivery. These cells might maintain reward representation over delays and facilitate encoding of associative representations in downstream brain regions such as BLA and nucleus accumbens (NAc) (Schoenbaum et al., 2009). lOFC damage may thus have disrupted associative signals for encoding delayed relative to immediate rewards.

\section{Medial OFC}

mOFC lesions decreased impulsive choice relative to both shamcontrols and lOFC lesions. These differences persisted during retraining after delay removal and are consistent with human neuroimaging studies. Preferential activation of $\mathrm{mOFC}$ is associated with choice of immediate primary or conditioned reinforcers relative to delayed ones (McClure et al., 2004, 2007) and correlates positively with individual differences in preference for smaller-immediate rewards on a delay-discounting task (Hariri et al., 2006). mOFC may thus participate in circuitry encoding associations between stimuli and/or responses that lead to immediate feedback of reward (Elliott et al., 2000; O'Doherty et al., 2001) and bias stimulus-driven, behavioral responses toward immediate goals (McClure et al., 2004, 2007). mOFC damage could disrupt such bias, shifting responding toward larger-delayed options.

Other researchers have hypothesized that mOFC may be better conceived as an integration site for subjective goal value signals (Kable and Glimcher, 2009), facilitating comparison of choice-options varying in parameters such as delay, magnitude, and probability. Under this framework, mOFC damage may affect delay-discounting by requiring adoption of choice strategies in the absence of such integrative value comparisons. Thus, mOFC-lesioned rats may have adopted a simpler, choice-bias strategy toward the larger reward (accounting less for withinsession changes in delays). This interpretation fits with reports that wOFC lesions lead to both increased delay-sensitivity and increased sensitivity to relative reward magnitudes (Kheramin et al., 2002). Indeed, our results further suggest that these processes may be anatomically dissociable: $1 \mathrm{OFC}$ lesions may preferentially mediate increases in delay-discounting (see Lateral OFC, above), whereas mOFC lesions may reduce delay-discounting preferentially through enhanced sensitivity to relative reward magnitudes. This interpretation also fits with evidence demonstrating increased progressive ratio break points in mOFC-lesioned mice (Gourley et al., 2010) as well as our observed facilitation of choice-preference following lever-position reversal in mOFClesioned rats, possibly driven by higher relative valuation of the large-reward option.

A recent study reported increased delay-discounting rates in patients with selective mOFC damage across three hypothetical intertemporal-choice tasks (Sellitto et al., 2010), in seeming contradiction to the present findings. However, given that their sequential choice-options varied in both delay and magnitude [quantitative models suggest such variations can potentially either suppress or promote delay-discounting (Kheramin et al., 2002)], a different choice strategy may have been adopted by patients with mOFC damage. Regardless of the precise interpretation, our results clearly implicate distinct functional contributions of the IOFC and mOFC in intertemporal choice performance. It would be of considerable interest to determine whether damage to these regions in human patients produce similarly opposing effects.

\section{Distinct and/or additive effects of OFC subregions}

Anatomical evidence in nonhuman primates shows that caudal lOFC has dense interconnections with NAc core, ventral tegmental area (VTA), and BLA, whereas mOFC has dense reciprocal connections with the hippocampus and only weak connectivity with NAc core, VTA, and BLA (Morecraft et al., 1992; Carmichael and Price, 1995). Recent studies in rodents also highlight the topographic separation of $\mathrm{mOFC}$ and lOFC projections to the striatum (Schilman et al., 2008).

These structural/functional distinctions are supported by our findings that $\mathrm{OFC}$ lesion size (i.e., $\mathrm{wOFC}>\mathrm{lOFC}>\mathrm{mOFC}$ ) is not related to the magnitude or direction of delay-discounting 
performance. Indeed, dissociable effects of lOFC and mOFC lesions may help explain wOFC-lesion effects. Behavioral effects of lOFC and mOFC lesions emerged at a time point remarkably similar to the loss of flattening of delay-discounting curves following wOFC lesions. Contrasting effects of lOFC and mOFC lesions may thus depend on experience-dependent (re)learning within the task and contribute distinctly and/or additively to delay-discounting (i.e., subregion effects cancelling out in wOFC rats). Moreover, contradictory findings among prior studies may result from variations in the duration of postsurgical assessment and the precise localization and extent of OFC lesions.

\section{Conclusions}

Using more discrete and selective lesions of medial and lateral OFC subregions, we demonstrate that lOFC lesions bias rats' choices toward small-immediate rewards, and mOFC lesions bias rats toward large-delayed rewards in a stable and dissociable manner from sham-operated controls. We thus provide the first evidence that the OFC is not unitary in its contribution to delaydiscounting in rodents.

\section{References}

Bechara A, Tranel D, Damasio H (2000) Characterization of the decisionmaking deficit of patients with ventromedial prefrontal cortex lesions. Brain 123:2189-2202.

Berlin HA, Rolls ET, Kischka U (2004) Impulsivity, time perception, emotion and reinforcement sensitivity in patients with orbitofrontal cortex lesions. Brain 127:1108-1126.

Bezzina G, Cheung TH, Asgari K, Hampson CL, Body S, Bradshaw CM, Szabadi E, Deakin JF, Anderson IM (2007) Effects of quinolinic acidinduced lesions of the nucleus accumbens core on inter-temporal choice: a quantitative analysis. Psychopharmacology (Berl.) 195:71-84.

Boettiger CA, Mitchell JM, Tavares VC, Robertson M, Joslyn G, D’Esposito M, Fields HL (2007) Immediate reward bias in humans: fronto-parietal networks and a role for the catechol-O-methyltransferase $158(\mathrm{Val} / \mathrm{Val})$ genotype. J Neurosci 27:14383-14391.

Boulougouris V, Dalley JW, Robbins TW (2007) Effects of orbitofrontal, infralimbic and prelimbic cortical lesions on serial spatial reversal learning in the rat. Behav Brain Res 179:219-228.

Cardinal RN, Aitken MR (2010) Whisker: a client-server high-performance multimedia research control system. Behav Res Methods 42:1059-1071.

Cardinal RN, Robbins TW, Everitt BJ (2000) The effects of d-amphetamine, chlordiazepoxide, alpha-flupenthixol and behavioural manipulations on choice of signalled and unsignalled delayed reinforcement in rats. Psychopharmacology (Berl.) 152:362-375.

Cardinal RN, Pennicott DR, Sugathapala CL, Robbins TW, Everitt BJ (2001) Impulsive choice induced in rats by lesions of the nucleus accumbens core. Science 292:2499-2501.

Carmichael ST, Price JL (1995) Limbic connections of the orbital and medial prefrontal cortex in macaque monkeys. J Comp Neurol 363:615-641.

Chudasama Y, Robbins TW (2003) Dissociable contributions of the orbitofrontal and infralimbic cortex to Pavlovian autoshaping and discrimination reversal learning: further evidence for the functional heterogeneity of the rodent frontal cortex. J Neurosci 23:8771-8780.

Doya K (2008) Modulators of decision making. Nat Neurosci 11:410-416.

Elliott R, Dolan RJ, Frith CD (2000) Dissociable functions in the medial and lateral orbitofrontal cortex: evidence from human neuroimaging studies. Cereb Cortex 10:308-317.

Fuchs RA, Evans KA, Parker MP, See RE (2004) Differential involvement of orbitofrontal cortex subregions in conditioned cue-induced and cocaineprimed reinstatement of cocaine seeking in rats. J Neurosci 24:6600-6610.

Gourley SL, Lee AS, Howell JL, Pittenger C, Taylor JR (2010) Dissociable regulation of instrumental action within mouse prefrontal cortex. Eur J Neurosci 32:1726-1734.

Hariri AR, Brown SM, Williamson DE, Flory JD, de Wit H, Manuck SB (2006) Preference for immediate over delayed rewards is associated with magnitude of ventral striatal activity. J Neurosci 26:13213-13217.
Hosmer DW, Lemeshow S (2000) Applied logisitic regression, 2nd edition. New York: Wiley.

Iversen SD, Mishkin M (1970) Perseverative interference in monkeys following selective lesions of the inferior prefrontal convexity. Exp Brain Res 11:376-386.

Kable JW, Glimcher PW (2009) The neurobiology of decision: consensus and controversy. Neuron 63:733-745.

Kheramin S, Body S, Mobini S, Ho MY, Velázquez-Martinez DN, Bradshaw CM, Szabadi E, Deakin JF, Anderson IM (2002) Effects of quinolinic acid-induced lesions of the orbital prefrontal cortex on inter-temporal choice: a quantitative analysis. Psychopharmacology (Berl.) 165:9-17.

Kringelbach ML, Rolls ET (2004) The functional neuroanatomy of the human orbitofrontal cortex: evidence from neuroimaging and neuropsychology. Prog Neurobiol 72:341-372.

Mar AC, Robbins TW (2007) Delay discounting and impulsive choice in the rat. Curr Protoc Neurosci Chapter 8: Unit 8.22.

McAlonan K, Brown VJ (2003) Orbital prefrontal cortex mediates reversal learning and not attentional set shifting in the rat. Behav Brain Res 146:97-103.

McClure SM, Laibson DI, Loewenstein G, Cohen JD (2004) Separate neural systems value immediate and delayed monetary rewards. Science 306:503-507.

McClure SM, Ericson KM, Laibson DI, Loewenstein G, Cohen JD (2007) Time discounting for primary rewards. J Neurosci 27:5796-5804.

Mobini S, Body S, Ho MY, Bradshaw CM, Szabadi E, Deakin JF, Anderson IM (2002) Effects of lesions of the orbitofrontal cortex on sensitivity to delayed and probabilistic reinforcement. Psychopharmacology (Berl.) 160:290-298.

Morecraft RJ, Geula C, Mesulam MM (1992) Cytoarchitecture and neural afferents of orbitofrontal cortex in the brain of the monkey. J Comp Neurol 323:341-358.

Murray EA, O'Doherty JP, Schoenbaum G (2007) What we know and do not know about the functions of the orbitofrontal cortex after 20 years of cross-species studies. J Neurosci 27:8166-8169.

O’Doherty J, Kringelbach ML, Rolls ET, Hornak J, Andrews C (2001) Abstract reward and punishment representations in the human orbitofrontal cortex. Nat Neurosci 4:95-102.

Paxinos G, Watson C (1998) The rat brain in stereotaxic co-ordinates, 4th ed. Sydney: Academic.

Roesch MR, Taylor AR, Schoenbaum G (2006) Encoding of timediscounted rewards in orbitofrontal cortex is independent of value representation. Neuron 51:509-520.

Rudebeck PH, Walton ME, Smyth AN, Bannerman DM, Rushworth MF (2006) Separate neural pathways process different decision costs. Nat Neurosci 9:1161-1168.

Schilman EA, Uylings HB, Galis-de Graaf Y, Joel D, Groenewegen HJ (2008) The orbital cortex in rats topographically projects to central parts of the caudate-putamen complex. Neurosci Lett 432:40-45.

Schoenbaum G, Roesch M (2005) Orbitofrontal cortex, associative learning, and expectancies. Neuron 47:633-636.

Schoenbaum G, Setlow B, Nugent SL, Saddoris MP, Gallagher M (2003) Lesions of orbitofrontal cortex and basolateral amygdala complex disrupt acquisition of odor-guided discriminations and reversals. Learn Mem 10:129-140.

Schoenbaum G, Roesch MR, Stalnaker TA, Takahashi YK (2009) A new perspective on the role of the orbitofrontal cortex in adaptive behaviour. Nat Rev Neurosci 10:885-892.

Sellitto M, Ciaramelli E, di Pellegrino G (2010) Myopic discounting of future rewards after medial orbitofrontal damage in humans. J Neurosci 30:16429-16436.

Wallis JD (2007) Orbitofrontal cortex and its contribution to decisionmaking. Annu Rev Neurosci 30:31-56.

Windmann S, Kirsch P, Mier D, Stark R, Walter B, Güntürkün O, Vaitl D (2006) On framing effects in decision making: linking lateral versus medial orbitofrontal cortex activation to choice outcome processing. J Cogn Neurosci 18:1198-1211.

Winstanley CA, Theobald DE, Cardinal RN, Robbins TW (2004) Contrasting roles of basolateral amygdala and orbitofrontal cortex in impulsive choice. J Neurosci 24:4718-4722. 